\title{
Dendrimers in Nanoscale Confinement: The Interplay between Conformational Change and Nanopore Entrance
}

\author{
Emel Ficici, ${ }^{\dagger}$ Ioan Andricioaei, ${ }^{* \dagger}$ and Stefan Howorka*, ${ }^{\star \dagger}$ \\ ${ }^{\dagger}$ Department of Chemistry, University of California Irvine, Irvine, California 92697, United States \\ ${ }^{\star}$ Department of Chemistry, Institute for Structural and Molecular Biology, University College London, London WC1H0AJ, England, \\ United Kingdom
}

\section{Supporting Information}

ABSTRACT: Hyperbranched dendrimers are nanocarriers for drugs, imaging agents, and catalysts. Their nanoscale confinement is of fundamental interest and occurs when dendrimers with bioactive payload block or pass biological nanochannels or when catalysts are entrapped in inorganic nanoporous support scaffolds. The molecular process of confinement and its effect on dendrimer conformations are, however, poorly understood. Here, we use single-molecule nanopore measurements and molecular dynamics simulations to establish an atomically detailed model of pore

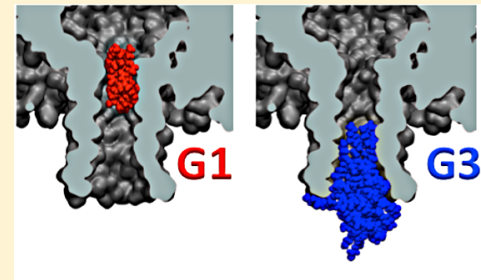

PAMAM Dendrimers

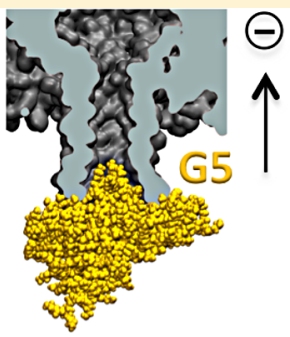
dendrimer interactions. We discover and explain that electrophoretic migration of polycationic PAMAM dendrimers into confined space is not dictated by the diameter of the branched molecules but by their size and generation-dependent compressibility. Differences in structural flexibility also rationalize the apparent anomaly that the experimental nanopore current read-out depends in nonlinear fashion on dendrimer size. Nanoscale confinement is inferred to reduce the protonation of the polycationic structures. Our model can likely be expanded to other dendrimers and be applied to improve the analysis of biophysical experiments, rationally design functional materials such as nanoporous filtration devices or nanoscale drug carriers that effectively pass biological pores.

KEYWORDS: nanopore, transport, dendrimer, molecular dynamics, single-molecule, ionization

$\mathrm{D}$ endrimers are nanoscale, star-like, hyperbranched molecules with high functional impact. ${ }^{1,2}$ Their structural hallmarks are (i) a void interior to encapsulate imaging agents or drugs, (ii) a dense outer shell with numerous terminal groups that can be chemically modified with fluorophores, catalytic groups, or molecular receptors, and (iii) a spherical shape that influences the interaction with other objects including nanopores. Biological nanopores help control molecular traffic across cellular membranes, whereas natural and man-made pores are exploited in nanotechnology for filtration $^{3}$ as well as the label-free sensing of analytes ${ }^{4-8}$ including electrical sequencing of individual DNA strands. ${ }^{9,10}$ Confining dendrimers temporary or permanently into nanopores is of relevance in basic and applied science. In research, permeating dendrimers of different size are used to probe the lumen of biological and inorganic nanopores, ${ }^{11}$ whereas in biomedicine, the interaction influences the transport of encapsulated therapeutics across biological membranes ${ }^{12,13}$ or achieves the blocking of pore-forming toxins. ${ }^{14,15}$ In materials science, the permanent lodging results in nanoscale filtration devices $^{16}$ and reusable catalysts that enclose the dendrimers within nanotubes. ${ }^{17}$

Despite these applications, a fundamental understanding of dendrimer behavior under nanoscale confinement is missing. For example, there is currently no definitive answer to the seemingly simple question about a cutoff size for the permeation of dendrimers into pores of given diameter, particularly under the ubiquitous transmembrane voltage present in biological cells as well as in research settings. Hence, it is not clear to which degree the structural flexibility of differently sized dendrimers influences their entrance into pores; their rigidity usually increases with bigger diameter. ${ }^{1}$ Furthermore, there is no detailed information on the mechanism for pore insertion, that is, whether the dendrimers insert completely or partly into a pore. Finally, it is not known whether the nanoscale confinement of dendrimers has an effect on the charge state of the ionizable functional groups of the molecules. A detailed molecular study that settles many of these questions will undoubtedly achieve a step-change in our fundamental understanding of dendrimer dynamics under confinement. Unfortunately, the large body of studies on the nanopore-mediated capturing or transport of linear organic polymers, DNA and polypeptides ${ }^{18-34}$ cannot be directly applied due to the different molecular shapes and properties compared to dendrimers.

Here, we develop a coherent and detailed molecular model of dendrimers under nanoscale confinement. We employ the widely used protein pore $\alpha$-hemolysin $(\alpha \mathrm{HL})$ (Figure 1A) of

Received: May 19, 2015

Revised: June 7, 2015 


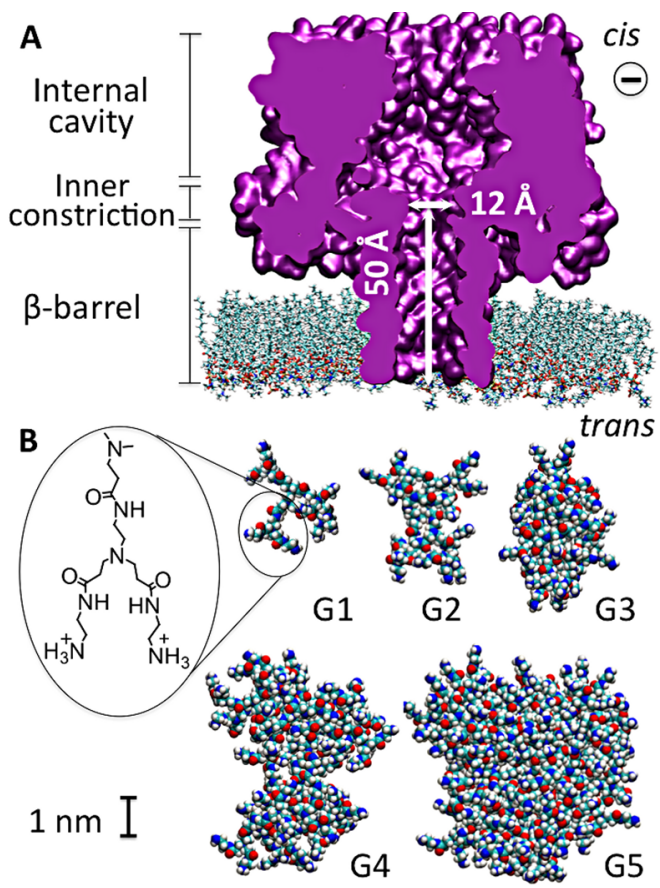

Figure 1. Structures of the molecular components used in our study. (A) Lipid membrane embedded $\alpha \mathrm{HL}$ pore featuring a transmembrane $\beta$-barrel of approximately $2 \mathrm{~nm}$ width and an $1.2 \mathrm{~nm}$-wide inner constriction. (B) PAMAM dendrimers of generations 1 to 5 . The inset shows the chemical structure of a PAMAM branch with protonated terminal primary amines. To study the interaction, dendrimers are placed on the trans side and an effective negative transmembrane potential is applied at the cis side to induce the electrophoretic movement of the dendrimers into the $\beta$-barrel.

known atomistic structure ${ }^{35}$ to examine the prominent polyamido-amine (PAMAM) dendrimers ${ }^{36}$ of increasing size, generation 1 to generation 5 (G1-G5) (Figure 1B; Supporting Information, Figure S-1). We measure the electrophoretically induced permeation of positively charged PAMAM at the level of individual pores and molecules, and thereby avoid problems of static or temporal heterogeneity that can hobble conventional ensemble measurements. ${ }^{37}$ The specific experimental output is the ionic current flowing through single pores as a function of PAMAM size. Our detailed experimental data are synergistically combined with molecular dynamics simulations to probe, at the atomic level, how far individual dendrimers permeate into the pore lumen. The simulations are validated by a comparison of the simulated to the experimental pore currents. Additionally, the simulations provide unprecedented molecular insight into the mechanism of PAMAM insertion, as well as the structural conformations and the charge of confined dendrimers as a function of their size and nanoscale confinement. These details could not be inferred solely from experiments. Our coherent understanding will help tailor the molecular properties of dendrimers and pores to suit specific applications.

Results and Discussion. Description of the Molecular Components of Our Study. In our work on nanoscale confinement, we examined whether PAMAM dendrimers G1 to G5 permeate into the $\alpha \mathrm{HL}$ pore. We selected these molecular components for three reasons. First, the size of the dendrimers is easily tunable. With each number of synthetic cycle (i.e., generation), twice the number of tertiary amine branching points are added, and the number of terminal primary amines doubles (Figure 1B; Supporting Information, Figure S-1). ${ }^{36}$ The hydrodynamic diameter of PAMAM thereby increases from $2.2 \mathrm{~nm}$ for G1 to $2.9 \mathrm{~nm}$ for $\mathrm{G} 2,3.6 \mathrm{~nm}$ for G3, $4.5 \mathrm{~nm}$ for G4, and $5.4 \mathrm{~nm}$ for G5 (Figure 1B). Second, the PAMAM dendrimer and the $\alpha \mathrm{HL}$ pore have matching nanoscale dimensions (Figure 1). ${ }^{35}$ Finally, the pore lumen at the trans side is of simple cylindrical shape, which was anticipated to facilitate studying the interaction with PAMAM. In particular, at the trans side, the $\alpha \mathrm{HL}$ pore features a cylinderlike transmembrane $\beta$-barrel of approximately $2 \mathrm{~nm}$ inner width and $5.0 \mathrm{~nm}$ height, which narrows to a $1.2 \mathrm{~nm}$-wide inner constriction (Figure 1A). The pore's cis side is geometrically less regular and has a sphere-like chamber and a 2.9-nm cis entrance (Figure 1A), which may cause further undesired complexity in our study of pore confinement. Hence, dendrimers were placed at the trans side.

Electrical Recordings of Single Pores Establish Dendrimer Permeation Patterns That Were Unexpected from Hydrodynamic Diameter Considerations Only. The generationdependent movement of PAMAM into the nanoscale confinement was probed by recording the ionic current flowing through single protein channels. Single-channel current recording is a widely used technique to understand the structural dynamics of individual molecules that are captured or passing the pore and can be examined in a label-free fashion under high temporal and conductance resolution. ${ }^{4-6}$ Our measurements with $\alpha \mathrm{HL}$ were carried out by applying a potential across a membrane with an embedded pore and by recording the ionic current of the solvated electrolytes. Under standard electrolyte conditions of $1 \mathrm{M} \mathrm{KCl}^{18,22,38}$ and an effective potential of -100 $\mathrm{mV}$ at the cis side, the blank $\alpha \mathrm{HL}$ pore exhibited a conductance of $1020 \pm 90 \mathrm{pS}$ ( $n=7$, number of independent recordings), which is in line with the literature. ${ }^{38-40}$ To probe dendrimers under nanoscale confinement, PAMAM were added at a final concentration of $50 \mu \mathrm{M}$ to the trans side of the $\alpha \mathrm{HL}$. Short current blockades were observed as exemplarily shown for PAMAM G2 (Figure 2A; Supporting Information, Figure S-2). The blockades are likely caused when the positively charged PAMAM is electrophoretically driven toward the negatively polarized cis side and temporarily resides within the $\beta$-barrel (Figure 1). The blockade ends when the PAMAM reversibly exits the pore likely to avoid the energetically unfavorable nanoscale confinement and to regain conformational and translational freedom. Remarkably, dendrimers of generation G1 to G4 entered the pore even though their hydrodynamic radius $(2.2 \mathrm{~nm}$ for $\mathrm{G} 1,4.5 \mathrm{~nm}$ for $\mathrm{G} 4)$ is larger than the transentrance of the $\beta$-barrel at $2.1 \mathrm{~nm}$. The diameter of the dendrimers is hence an unsuitable guide to predict their electrophoretic movement into a narrow pore.

Nonmonotonous Dependence of Current Blockade on Dendrimer Generation. To further examine the permeation of large dendrimers, pore blockades were characterized in terms of their blockade amplitude, $A$, and duration, $\tau_{\text {off }}$ (Figure 2B). The amplitude is the difference between the ionic current at the bottom of the event, $I_{\mathrm{b}}$, and the current of the open channel, $I_{\mathrm{o}}$, normalized to $I_{\mathrm{o}}$ (Figure $2 \mathrm{~B}$ ). To obtain statistically relevant data, the distribution of $A$ was plotted in a histogram as exemplarily shown for the G2 dendrimer (Figure 2C). The peak of the distribution has a maximum of $46 \%$. The wide distribution of amplitude values in the histogram might reflect the known polydispersity of PAMAM dendrimers. In HPLC analysis of the commercially sourced dendrimer sample, ${ }^{16}$ we found a broad and single major peak that confirms the 
A

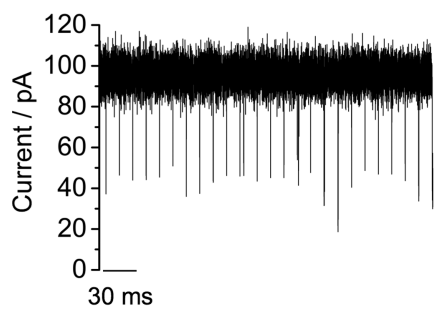

C

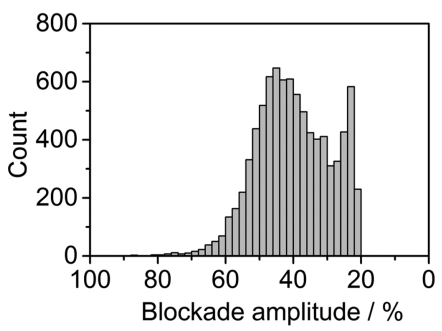

B

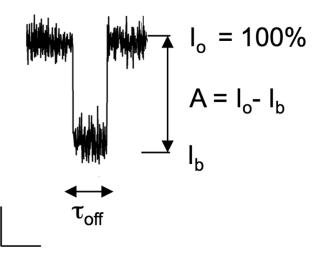

D

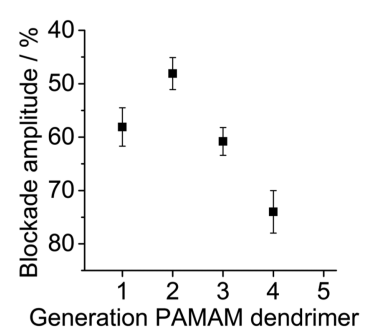

Figure 2. PAMAM dendrimers temporarily block the $\alpha \mathrm{HL}$ pore with large dendrimer diameter and in an unusual nonlinear dependence on generation. (A) Current blockades caused by PAMAM G2 added to the trans side of $\alpha \mathrm{HL}$ recorded at $1 \mathrm{M} \mathrm{KCl}$, at an effective potential of $-100 \mathrm{mV}$ at the cis pore side. (B) Single blockade event with duration $\tau_{\text {off }}$ and amplitude $A$, defined by the difference between the openchannel current $I_{\mathrm{o}}$ and the current level for the blocked channel, $I_{\mathrm{b}}$. (C) Histogram of amplitude $A$ with a peak at $46 \%$. The peak at $22 \%$ stems from short noise fluctuations of the open channel and was included to avoid the artificial trimming of the main blockade peak. The drop at $20 \%$ in the histogram reflects the cut-off threshold used for detecting events in the current recordings. (D) Blockade amplitude depends in a nonmonotonous fashion on the dendrimer generation.

polydispersity but argues against heterogeneities. The average blockade amplitude for each PAMAM generation was acquired using at least three independent recordings with a total of around 10000 individual events. No correlation was detected between the width of the amplitude distribution in histograms and the PAMAM generation even though the latter scales with polydispersity. ${ }^{1}$ Likely, this implies that the interaction with higher-generation dendrimers does not involve the complete PAMAM molecule, as established further below.

A plot of average maximum peak amplitude vs dendrimer generation (Figure 2D) summarizes the results. Several remarkable features are noted. First, the plot shows an unusual nonlinear dependence of $A$ vs dendrimer generation. The amplitude of the current blockade first lowers from $58.1 \pm 3.6 \%$ (G1) to $48.1 \pm 3.0 \%$ (G2) before increasing to over $60.8 \pm$ $2.8 \%$ (G3) and $74 \pm 4.4 \%$ (G4). This behavior is independent of the transmembrane voltage (Supporting Information, Figures S-3 and S-4) and likely implies that the dendrimers lodged in the pore are not in a metastable state but at the energetic minimum. Second, G4 PAMAM migrated into the pore only at very high potentials of $160 \mathrm{mV}$. This can suggest that the lower voltages were not enough to force the dendrimer into the pore. Finally, G5 PAMAM was not electrophoresed into the pore, as judged by the lack of discernible clear current blockades.

When considering dendrimers G1 to G3, the blockade duration, $\tau_{\text {off }}$ depended in nonlinear fashion on the dendrimer size (Supporting Information, Figure S-5), similar to the amplitude, whereas it was not influenced by voltage (Supporting Information, Figure S-3B). We did not pursue

the analysis of $\tau_{\text {off }}$ data any further, as we were able to computationally simulate the blockade amplitude.

Molecular Dynamics Simulations of PAMAM Permeation into $\alpha H L$. In order to explain the unusual blockade of highgeneration dendrimers and the nonmonotonous dependence of blockade amplitude, we atomistically simulated the electrophoretically driven permeation of PAMAM into $\alpha \mathrm{HL}$ using molecular dynamics. We first modeled the dendrimers ${ }^{41}$ and pre-equilibrated them for $30 \mathrm{~ns}$. Representative structures are shown in Figure 3, and the convergence of radii of gyration is
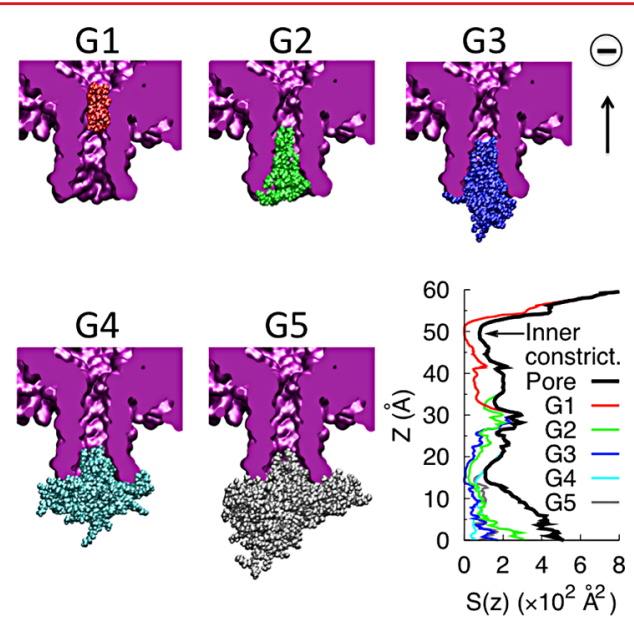

Figure 3. Snapshots of PAMAM dendrimers inserted into the $\beta$-barrel of $\alpha \mathrm{HL}$ after molecular dynamics simulations for electrophoresis into the pore, as implied by the arrow at the right. The dendrimer generations are indicated. In the bottom right is a plot of the open cross-sectional area $\left(S^{\prime}(z)\right)$ whereby the $z$-axis position is $0 \AA$ at the trans entrance of the $\beta$-barrel and $49.5 \AA$ at the inner constriction. The membrane is not shown for reasons of visual clarity.

demonstrated in Supporting Information, Figure S-6. The dendrimer radii were found to be $9.7,12.1,13.3,18.7$, and 21.0 $\AA$ for G1 through G5, respectively, within the range of previously published values. ${ }^{42}$ It has been shown that at $\mathrm{pH}$ values between 7 and 8, only dendrimer primary amines are protonated. $^{43,44}$ Therefore, we used this convention in modeling the dendrimers at neutral $\mathrm{pH}$ used in the experiment. The dendrimers were then added to the trans side of $\alpha \mathrm{HL}$ pore at a distance equivalent to twice the radius of gyration of dendrimer. The protein pore was embedded in a preequilibrated, circular patch of a lipid DPPC leaflet with a radius of $60 \AA$. In order to reduce the computation time while maintaining the key interactions, all atoms were fixed except for the dendrimer and the charged $\alpha \mathrm{HL}$ residues at the pore entrance, that is, ASP127 and LYS131 on each of the seven monomers near $z=0 \AA$, which coindices with the opening of the $\beta$-barrel. Solvent was represented with a continuum model by using generalized Born implicit solvent as implemented in NAMD. ${ }^{45,46}$ All simulations were run by using NAMD software $^{46}$ with the CHARMM27 force field. ${ }^{47}$

The computational electrophoresis of the positively charged PAMAM dendrimers was achieved by applying a transmembrane potential following an established protocol ${ }^{48,49}$ and grid-steered molecular dynamics (GSMD). ${ }^{50}$ In this approach, an electrostatic grid potential obtained at low voltages is scaled up to accelerate the molecular movement to times scales accessible to simulations while reducing artificial distortions in molecular configurations. ${ }^{50}$ GSMD has been 
successfully applied to simulate the translocation of DNA strands, DNA hairpins and $\alpha$-helical peptides through $\alpha \mathrm{HL}{ }^{50}$ In our simulations, we initially used an effective voltage of $30 \mathrm{~V}$ for $500 \mathrm{ps}$, only $12 \mathrm{~V}$ of which is due to the contribution from constant electric field. We then equilibrated the structure at a lower effective voltage of $4.8 \mathrm{~V}$ for $1 \mathrm{~ns}$ and finally $0.5 \mathrm{~ns}$ at a potential of $120 \mathrm{mV}$ for $0.5 \mathrm{~ns}$ to obtain meaningful conformations. Further details of the GSMD settings are provided in the Supporting Information.

Molecular Dynamics Establish That Pore Permeation Is Not Directly Determined by Dendrimer Diameter but by the Generation-Dependent Compressibility of PAMAM. The results of our simulations are summarized in Figure 3. They reveal that the permeation into the pore strongly depends on the dendrimer generation. G1 completely entered the $\beta$-barrel to block the inner constriction, whereas G2 did not reach as deeply. By contrast, G3 did not fully enter the barrel, and only a smaller portion of G4 and G5 was inside the pore.

The simulations are highly valuable as they first replicate the experimentally found permeation of large dendrimers. Similar to nanopore recordings, all PAMAM dendrimers from G1 to G4 at least partly if not completely entered the pore in the simulations (Figure 3) even though the hydrodynamic diameter for all is larger than the trans entrance. Second, the computational data distinguish two molecular modes for pore entrance. G1 and G2 enter the pore completely (Figure 3). By contrast, only several branches of the dendrimer G4 are able to reach into the pore lumen (Figure 3). G3 occupies a middle position between the two extremes. The simulations, third, provide a reason for the generation-dependent entrance mode in terms of structural flexibility and compressibility of the outermost dendrimer shell that decreases with higher generation. ${ }^{51}$ The different degrees of flexibilities are apparent in the computationally derived dendrimers' terminal relative mobilities which represent the size-dependent change in the angle spanned from the dendrimer center by two terminal branches (Supporting Information Figures S-9 and S-10). As a further illustration that rigidity increases with dendrimer size, we show in Supporting Information Figures S-11 and S-12 that the intramolecular potential energy required to push the dendrimer into the pore scales with generation.
Using this insight, we can explain that G1 and G2 can enter so deeply into the pore because the required associated rearrangement of the PAMAM branches is energetically feasible due to the flexible and noncrowded outer dendrimer shell. By contrast, only a few branches of the G4 dendrimer (Figure 3) enter the pore because the crowded outer shell of the dendrimer does not allow for a larger structural reorganization as found for G1 or G2. Indeed, G4 cannot reach deeper into the pore as this would require the energetically costly pushing aside the other branches of the dendrimer. One discrepancy is that PAMAM G5 entered the pore in the simulations but not in the experiments. A possible reason for this mismatch could be that a PAMAM G5 branch resides too short in the barrel to be detected amid the current noise of the open channel.

To achieve a quantitative measure of the varying degrees of pore blockade, we computed from the molecular dynamics simulations the open cross-sectional area of the lumen, $S(z)$, against the $z$ coordinate running along the central pore axis (Figure 3). $S(z)$ is the area of the pore which is not blocked by a PAMAM dendrimer. For reference, the open (non-PAMAM blocked) pore area is in black (Figure 3). The plot highlights that G1 blocks the pore at the inner constriction while G2 and G3 constrict mostly the middle part of the $\beta$-barrel.

Simulations Allow Us to Calculate the lonic Current Blockade and Thereby Explain the Unusual Experimental Nonmonotonous Dependence of Blockade Amplitude on Dendrimer Generation. The open cross-sectional area $S(z)$ of the lumen was used to calculate the ionic current blockade. $S(z)$ integrated over $z$ represents the volume of pore lumen which is filled by electrolytes, which is in turn directly proportional to the ionic current. Although more sophisticated means to compute current by representing ions explicitly exists, ${ }^{49,52,53}$ they are computationally more challenging to converge because of the direct calculation of the timedependent flow of ions, particularly for large ionic flows. Moreover, because the current are so large for the system at hand, the approximation we use is expected to be appropriate. Following our previous work, ${ }^{54}$ the ratio of blocked channel, $I_{\mathrm{b}}$ and the open-channel current $I_{\mathrm{o}}$ was calculated using

$$
\frac{I_{\mathrm{b}}}{I_{\mathrm{o}}}=\frac{\int_{0}^{L} \frac{\mathrm{d} z}{S(z)}+\left(\frac{1}{2 d_{1}}+\frac{1}{2 d_{2}}\right)}{\int_{0}^{L} \frac{\mathrm{d} z}{S^{\prime}(z)}+\delta_{1,2}\left(\frac{1}{2 d_{1}^{\prime}}\right)+\delta_{3,4,5}\left(\rho\left(\frac{1}{2 d_{1}^{\prime}}-\frac{1}{\pi D}\right)+\frac{1}{\pi D}\right)+\left(\frac{1}{2 d_{2}}\right)}
$$

Here, $S(z)$ and $S^{\prime}(z)$ in the numerator and denominator are the open cross-sectional areas when the pore is open and when the pore is dendrimer-blocked, respectively. The terms $1 /\left(2 d_{1}\right)$ and $1 /\left(2 d_{2}\right)$ in the numerator are the access resistances for the trans and cis pore opening of the unblocked channel with diameters $d_{1}$ and $d_{2}$, respectively, following Hall's model. ${ }^{55}$

The trans access resistance of pores blocked by G1 and G2 was calculated similarly using the effective pore diameter $d_{1}^{\prime}$ at the trans side. A different approach was used to describe the access resistance of dendrimers G3, G4, and G5, which due to their larger size reside to an increasing degree outside the pore, within a roughly hemispherical region. In our calculations, we treated the region outside the pore as a combination of (i) a hemisphere with a diameter $D$ large enough to enclose the dendrimer partition outside the pore and (ii) the rest of the region from this hemisphere up to the electrode at infinity. The contribution to access resistance from the latter term is given by $(1 / \pi D)$ as described in ref. ${ }^{56}$ In the region from the surface of this hypothetical hemisphere up to the PAMAM-blocked pore entrance of effective diameter of $d_{1}^{\prime}$, the access resistance can be calculated by the term $\rho\left(\left(1 /\left(2 d_{1}^{\prime}\right)\right)-(1 /(\pi D))\right)$, where $\rho$ is the effective resistivity introduced by the dendrimer, a relative resistivity normalized with respect to the resistivity of the pure solution. As a result, the total relative access resistance in the trans side space is $\rho\left(\left(1 /\left(2 d_{1}^{\prime}\right)\right)-(1 /(\pi D))\right)+(1 /(\pi D))$. The treatment of access resistance for different dendrimer generations can be summarized as follows: 


$$
\begin{aligned}
& \delta_{1,2}= \begin{cases}1, & \text { for } \mathrm{G} 1 \text { and } \mathrm{G} 2 \\
0, & \text { otherwise }\end{cases} \\
& \delta_{3,4,5}= \begin{cases}1, & \text { for } \mathrm{G} 3, \mathrm{G} 4, \text { and } \mathrm{G} 5 \\
0, & \text { otherwise }\end{cases}
\end{aligned}
$$

The parameter $\rho$, accounting for the relative increase in resistivity caused by PAMAM outside the pore region, can be further factored out into two components, $\rho=\rho_{1}\left(V, V^{\prime}\right) \rho_{2}(q)$, that is, into a steric and an electrostatic factor to mirror the reduction in free solubilized electrolyte ions within the region. The steric component is deduced from the reduction of the solvent accessible (free) volume by the dendrimer, and a linear relationship between electrolyte concentration and resistivity was assumed from a first-order expansion of the Kohlrausch law. ${ }^{57}$ This leads to an expression for the steric relative resistivity $\rho_{1}=V / V^{\prime}$, where $V$ is the volume of a hemisphere with diameter $D$ and $V^{\prime}$ is the solvent accessible portion of this volume in the presence of dendrimer. The second component $\rho_{2}$ reflects the lowering of electrolyte concentration caused by the electrostatic repulsion against the charged, protonated PAMAM terminal amine groups positioned outside the pore and is assumed to increase linearly with the number of charges. The protonation in this structurally noncompressed PAMAM parts differs to the dendrimer branches which are confined within the pore. There, the protonation of their terminal primary amines is, in agreement with other polyelectrolytes, ${ }^{58,59}$ assumed to be suppressed to avoid mutual energetically unfavorable electrostatic repulsion, as reflected by the solely steric term $S^{\prime}(z)$ for calculating the ionic current. The Supporting Information provides a more detailed description of how the values of the parameter $\rho$ were calculated.

The blockade amplitude was deduced from the ratio of $I_{\mathrm{b}} / I_{\mathrm{o}}$ using eq 4

$$
\% \text { blockade amplitude }=100 \times\left(1-\frac{I_{\mathrm{b}}}{I_{\mathrm{o}}}\right)
$$

As shown in Figure 4, the theory was able to capture well the experimentally found nonmonotonous dependence of pore

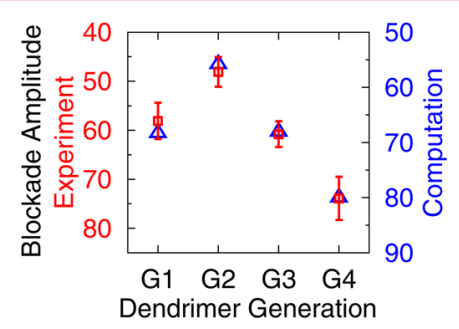

Figure 4. Comparison of experimental (red) and computational (blue) blockade amplitude caused by the permeation of PAMAM into the $\alpha \mathrm{HL}$ pore.

blockade on dendrimer generation. This is of note, as our model does not use a phenomenological fit to experimental data but rather calculates the current from dendrimer-induced and position-dependent blocked pore and access volumes and the electrostatic factors. The small offset of approximately $10 \%$ between the computational and experimental blockade levels could have been overcome by including a mathematical fitting factor in eq 1, but doing so would have diluted the simplicity and biophysical coherence of our model. The computational model correctly predicts the experimentally observed trend, yet it slightly and consistently overestimates the blockade amplitude. A possible explanation for this consistent shift could be the different time scales underlying the simulated dynamics vs the experimental measurement of the poreblocking events. As the temporal resolution of the measurement is about $50 \mu \mathrm{s}$, time-averaged partially blocked conformations could be present in the measured data, but not in the simulation, which accounts for nanosecond dynamics in the thermodynamically dominant state of dendrimer-pore interactions.

Nevertheless, the agreement between theory and experiment is remarkable considering that the model is relatively simple compared to several more complex computational strategies. ${ }^{49,52,53}$ A key reason contributing to the agreement, particularly for G3 and G4, has to do with the relative resistivities of PAMAM inside and outside the channel. The difference in the resistivities is biophysically justified by the steric component, and by the fact that squeezing PAMAM inside the channel pushes charged terminal amino groups together and hence lowers their protonation state to avoid energetically unfavorable electrostatic repulsion under confinement. Consequently, PAMAM branches inside the pore are considered to solely act as steric but not as an electrostatic barrier for the passage of electrolyte cations, which in turn results in a higher ionic current compared to flux through PAMAM outside the pore. When expressed via the parameter $\rho$, the resistivity of nonconfined PAMAM is about four times bigger for G3 and 21 times bigger for G4 than of dendrimer branches confined inside the pore. This parameter is key for obtaining a good match to the data, as setting it to equal in and outside the pore resulted in a strong mismatch between experiment and data as shown by a comparison of Supporting Information Figure S-7 and Figure S-8.

Conclusions. We have measured and simulated how PAMAM dendrimers permeate into the widely used model pore $\alpha \mathrm{HL}$ to uncover fundamental insight into dendrimerpore interactions at the nanoscale. The key findings of our study are threefold. First, we show that permeation is not governed by the apparent hydrodynamic size of the dendrimers relative to the pore. Rather, it is determined by their generation-dependent conformational flexibility, that is, by the details of if and how dendrimers can modulate their internal structure to enter the pore. The result is important for basic and applied science as it is now easier to predict the optimal dendrimer generation for a given nanopore diameter. Second, our data suggest that pore-confinement is reducing the ionization state of PAMAM, which in turn can help predict permeation properties through charged nanopores. Third, we demonstrate that the permeation-induced current blockade exhibits a nontrivial dependence on dendrimer generation. This is caused by an intriguing interplay pore geometry, different dendrimer compressibilities, and confinement-induced changes in PAMAM properties, including ionization. Small, easily compressible dendrimers like G1 and G2 completely move into the cylinder-shaped pore to cause a large blockage caused by steric factors. By contrast, at the other extreme, larger, less compressible dendrimer G4 does not completely enter the pore but causes a larger current blockade as the PAMAM section outside the pore is fully protonated to electrostatically reduce electrolyte mobility. In conclusion, our study highlights the importance of molecular flexibility and nontrivial nanoparticle nanopore interactions in modulating pore transport and can 
likely be expanded to other dendrimers or molecules designed to permeate or lodge inside nanoporous structures to improve applications in biotechnology, biomedicine, material science, and biophysical research.

\section{ASSOCIATED CONTENT}

\section{S Supporting Information}

Detailed description of current blockade calculations and supplementary results, additional analysis of translocation events, and simulation trajectory movies. The Supporting Information is available free of charge on the ACS Publications website at DOI: 10.1021/acs.nanolett.5b01960.

\section{AUTHOR INFORMATION}

\section{Corresponding Authors}

*E-mail: andricio@uci.edu.

*E-mail: s.howorka@ucl.ac.uk.

\section{Notes}

The authors declare no competing financial interest.

\section{ACKNOWLEDGMENTS}

The work was supported by the Engineering and Physical Science Research Council (\#08000459) and the Department of Chemistry at University College London. The research used resources of the National Energy Research Scientific Computing Center, a DOE Office of Science User Facility supported by the Office of Science of the U.S. Department of Energy under Contract No. DE-AC02-05CH11231. This work also used the Extreme Science and Engineering Discovery Environment (XSEDE), which is supported by National Science Foundation grant number ACI-1053575. We thank Dr. Aleksei Aksimentiev for providing files to conduct grid-steered MD. We also thank Dr. Zuzanna Siwy for helpful discussions and Dr. Mahua Roy for providing code which was adapted for current blockade calculations.

\section{REFERENCES}

(1) Astruc, D.; Boisselier, E.; Ornelas, C. Chem. Rev. 2010, 110 (4), $1857-1959$

(2) Lee, C. C.; MacKay, J. A.; Frechet, J. M.; Szoka, F. C. Nat. Biotechnol. 2005, 23 (12), 1517-1526.

(3) Baker, L. A.; Bird, S. P. Nat. Nanotechnol. 2008, 3 (2), 73-74.

(4) Howorka, S.; Siwy, Z. Chem. Soc. Rev. 2009, 38 (8), 2360-2384.

(5) Bayley, H.; Cremer, P. S. Nature 2001, 413 (6852), 226-230.

(6) Mayer, M.; Yang, J. Acc. Chem. Res. 2013, 46 (12), 2998-3008.

(7) Stoloff, D. H.; Wanunu, M. Curr. Opin. Biotechnol. 2013, 24 (4), 699-704.

(8) Perera, R. T.; Fleming, A. M.; Johnson, R. P.; Burrows, C. J.; White, H. S. Nanotechnology 2015, 26 (7), 074002.

(9) Cherf, G. M.; Lieberman, K. R.; Rashid, H.; Lam, C. E.; Karplus, K.; Akeson, M. Nat. Biotechnol. 2012, 30 (4), 344-348.

(10) Manrao, E. A.; Derrington, I. M.; Laszlo, A. H.; Langford, K. W.; Hopper, M. K.; Gillgren, N.; Pavlenok, M.; Niederweis, M.; Gundlach, J. H. Nat. Biotechnol. 2012, 30 (4), 349-353.

(11) Ignacio-de Leon, P. A. A.; Zharov, I. Chem. Commun. 2011, 47 (1), 553-555.

(12) Sarin, H.; Kanevsky, A. S.; Wu, H.; Brimacombe, K. R.; Fung, S. H.; Sousa, A. A.; Auh, S.; Wilson, C. M.; Sharma, K.; Aronova, M. A.; Leapman, R. D.; Griffiths, G. L.; Hall, M. D. J. Transl. Med. 2008, 6, 80.

(13) Sarin, H.; Kanevsky, A. S.; Wu, H.; Sousa, A. A.; Wilson, C. M.; Aronova, M. A.; Griffiths, G. L.; Leapman, R. D.; Vo, H. Q. J. Transl. Med. 2009, 7, 51.

(14) Kong, L.; Harrington, L.; Li, Q.; Cheley, S.; Davis, B. G.; Bayley, H. Nat. Chemistry 2013, 5 (8), 651-659.
(15) Forstner, P.; Bayer, F.; Kalu, N.; Felsen, S.; Fortsch, C.; Aloufi, A.; Ng, D. Y.; Weil, T.; Nestorovich, E. M.; Barth, H. Biomacromolecules 2014, 15 (7), 2461-2474.

(16) Martin, H.; Kinns, H.; Mitchell, N.; Astier, Y.; Madathil, R.; Howorka, S. J. Am. Chem. Soc. 2007, 129 (31), 9640-9649.

(17) Lindner, J. P.; Roben, C.; Studer, A.; Stasiak, M.; Ronge, R.; Greiner, A.; Wendorff, H. J. Angew. Chem., Int. Ed. 2009, 48 (47), 8874-8877.

(18) Kasianowicz, J. J.; Brandin, E.; Branton, D.; Deamer, D. W. Proc. Natl. Acad. Sci. U.S.A. 1996, 93 (24), 13770-13773.

(19) Vercoutere, W.; Akeson, M. Curr. Opin. Chem. Biol. 2002, 6 (6), $816-822$.

(20) Deamer, D. W.; Branton, D. Acc. Chem. Res. 2002, 35 (10), $817-825$.

(21) Maglia, G.; Restrepo, M. R.; Mikhailova, E.; Bayley, H. Proc. Natl. Acad. Sci. U.S.A. 2008, 105 (50), 19720-19725.

(22) Butler, T. Z.; Gundlach, J. H.; Troll, M. A. Biophys. J. 2006, 90 (1), 190-199.

(23) Wang, H.; Dunning, J. E.; Huang, A. P.; Nyamwanda, J. A.; Branton, D. Proc. Natl. Acad. Sci. U.S.A. 2004, 101 (37), 13472-13477.

(24) Wanunu, M.; Chakrabarti, B.; Mathe, J.; Nelson, D. R.; Meller, A. Phys. Rev. E 2008, 77 (3), 031904.

(25) Wiggin, M.; Tropini, C.; Tabard-Cossa, V.; Jetha, N. N.; Marziali, A. Biophys. J. 2008, 95 (11), 5317-5323.

(26) Meller, A.; Nivon, L.; Branton, D. Phys. Rev. Lett. 2001, 86 (15), $3435-3438$.

(27) Howorka, S.; Bayley, H. Biophys. J. 2002, 83 (6), 3202-3210.

(28) Sutherland, T. C.; Long, Y. T.; Stefureac, R.; Bediako-Amoa, I.; Kraatz, H. B.; Lee, J. S. Nano Lett. 2004, 4 (7), 1273-1277.

(29) Movileanu, L.; Schmittschmitt, J. P.; Scholtz, J. M.; Bayley, H. Biophys. J. 2005, 89 (2), 1030-1045.

(30) Stefureac, R.; Long, Y. T.; Kraatz, H. B.; Howard, P.; Lee, J. S. Biochemistry 2006, 45 (30), 9172-9179.

(31) Huang, L.; Kirmizialtin, S.; Makarov, D. E. J. Phys. Chem. B 2005, 123 (12), 124903.

(32) Oukhaled, G.; Mathé, J.; Biance, A. L.; Bacri, L.; Betton, J. M.; Lairez, D.; Pelta, J.; Auvray, L. Phys. Rev. Lett. 2007, 98 (15), 158101.

(33) Talaga, D. S.; Li, J. J. Am. Chem. Soc. 2009, 131 (26), 92879297.

(34) Franceschini, L.; Soskine, M.; Biesemans, A.; Maglia, G. Nat. Commun. 2013, 4, 2415.

(35) Song, L.; Hobaugh, M. R.; Shustak, C.; Cheley, S.; Bayley, H.; Gouaux, J. E. Science 1996, 274 (5294), 1859-1866.

(36) Bauer, B. J.; Amis, E. J. Characterization of dendritically branched polymers by small angle neutron scattering (SANS), small angle X-ray scattering (SAXS) and transmission electron microscopy (TEM). In Dendrimers and other dendritic polymers; Frechet, J. M., Tomalia, D. A., Eds. John Wiley \& Sons: Hoboken, NJ, 2002; pp 255284.

(37) Zlatanova, J.; van Holde, K. Mol. Cell 2006, 24 (3), 317-329.

(38) Mitchell, N.; Howorka, S. Angew. Chem., Int. Ed. 2008, 47 (30), 5476-5479.

(39) Howorka, S.; Movileanu, L.; Braha, O.; Bayley, H. Proc. Natl. Acad. Sci. U.S.A. 2001, 98 (23), 12996-13001.

(40) Howorka, S.; Cheley, S.; Bayley, H. Nat. Biotechnol. 2001, 19 (7), 636-639.

(41) Kelly, C. V.; Leroueil, P. R.; Nett, E. K.; Wereszczynski, J. M.; Baker, J. R.; Orr, B. G.; Holl, M. M. B.; Andricioaei, I. J. Phys. Chem. B 2008, 112 (31), 9337-9345.

(42) Maiti, P. K.; Cagin, T.; Wang, G. F.; Goddard, W. A. Macromolecules 2004, 37 (16), 6236-6254.

(43) Cakara, D.; Kleimann, J.; Borkovec, M. Macromolecules 2003, 36 (11), 4201-4207.

(44) Lee, I.; Athey, B. D.; Wetzel, A. W.; Meixner, W.; Baker, J. R. Macromolecules 2002, 35 (11), 4510-4520.

(45) Tanner, D. E.; Chan, K. Y.; Phillips, J. C.; Schulten, K. J. Chem. Theory Comput. 2011, 7 (11), 3635-3642. 
(46) Phillips, J. C.; Braun, R.; Wang, W.; Gumbart, J.; Tajkhorshid, E.; Villa, E.; Chipot, C.; Skeel, R. D.; Kale, L.; Schulten, K. J. Comput. Chem. 2005, 26 (16), 1781-1802.

(47) MacKerell, A. D.; Bashford, D.; Bellott, M.; Dunbrack, R. L.; Evanseck, J. D.; Field, M. J.; Fischer, S.; Gao, J.; Guo, H.; Ha, S.; Joseph-McCarthy, D.; Kuchnir, L.; Kuczera, K.; Lau, F. T. K.; Mattos, C.; Michnick, S.; Ngo, T.; Nguyen, D. T.; Prodhom, B.; Reiher, W. E.; Roux, B.; Schlenkrich, M.; Smith, J. C.; Stote, R.; Straub, J.; Watanabe, M.; Wiorkiewicz-Kuczera, J.; Yin, D.; Karplus, M. J. Phys. Chem. B 1998, 102 (18), 3586-3616.

(48) Roux, B. Biophys. J. 2008, 95 (9), 4205-4216.

(49) Aksimentiev, A.; Schulten, K. Biophys. J. 2005, 88 (6), 37453761.

(50) Wells, D. B.; Abramkina, V.; Aksimentiev, A. J. Chem. Phys. 2007, 127 (12), 125101.

(51) Hierlemann, A.; Campbell, J. K.; Baker, L. A.; Crooks, R. M.; Ricco, A. J. J. Am. Chem. Soc. 1998, 120 (21), 5323-5324.

(52) Noskov, S. Y.; Im, W.; Roux, B. Biophys. J. 2004, 87 (4), 22992309

(53) Freites, J. A.; Schow, E. V.; White, S. H.; Tobias, D. J. Biophys. J. 2012, 102 (11), A44-A46.

(54) Mereuta, L.; Roy, M.; Asandei, A.; Lee, J. K.; Park, Y.; Andricioaei, I.; Luchian, T. Sci. Rep. 2014, 4, 3885.

(55) Hall, J. E. J. Gen. Physiol. 1975, 66 (4), 531-532.

(56) Hille, B. Prog. Biophys. Mol. Biol. 1970, 21, 1-32.

(57) Atkins, P.; De Paula, J. Atkins' Physical Chemistry; Oxford University Press: Oxford, U.K., 2006.

(58) Nap, R. J.; Tagliazucchi, M.; Szleifer, I. J. Chem. Phys. 2014, 140 (2), 024910

(59) Longo, G. S.; de la Cruz, M. O.; Szleifer, I. Langmuir 2014, 30 (50), 15335-15344. 